\title{
A CHAIN RULE FOR ESSENTIALLY SMOOTH LIPSCHITZ FUNCTIONS*
}

\author{
JONATHAN M. BORWEIN ${ }^{\dagger}$ AND WARREN B. MOORS $\ddagger$
}

\begin{abstract}
In this paper we introduce a new class of real-valued locally Lipschitz functions (that are similar in nature and definition to Valadier's saine functions), which we call arcwise essentially smooth, and we show that if $g: R^{m} \rightarrow R$ is arcwise essentially smooth on $R^{m}$ and each function $f_{j}: R^{n} \rightarrow R, 1 \leq j \leq m$, is strictly differentiable almost everywhere in $R^{n}$, then $g \circ f$ is strictly differentiable almost everywhere in $R^{n}$, where $f \equiv\left(f_{1}, f_{2}, \ldots, f_{m}\right)$. We also show that all the semismooth and all the pseudoregular functions are arcwise essentially smooth. Thus, we provide a large and robust lattice algebra of Lipschitz functions whose generalized derivatives are well behaved.
\end{abstract}

Key words. Lipschitz functions, chain rule, Haar-null sets, differentiability, essentially smooth

AMS subject classifications. Primary, 49J520, 46N10; Secondary, 58C20

PII. S1052623496297838

1. Introduction. In this paper we show that those Lipschitz functions which are strictly differentiable almost everywhere (that is, essentially smooth) possess extremely strong closure properties. Essentially smooth functions were studied in great detail in [3] and [4]. In particular, it was shown therein that all such functions possess very well-behaved Clarke generalized gradients.

We begin by recalling some preliminary definitions regarding the Clarke subdifferential mapping [6]. A real-valued function $f$ defined on a nonempty open subset $U$ of a Banach space $X$ is locally Lipschitz on $U$ if for each $x_{0} \in U$ there exists a $K>0$ and a $\delta>0$ such that

$$
|f(x)-f(y)| \leq K\|x-y\| \quad \text { for all } \quad x, y \in B\left(x_{0}, \delta\right) .
$$

For functions in this class, it is often instructive to consider the following three directional derivatives:

(i) The upper Dini derivative at $x \in U$, in the direction $y$, is given by

$$
f^{+}(x ; y) \equiv \limsup _{\lambda \rightarrow 0^{+}} \frac{f(x+\lambda y)-f(x)}{\lambda} .
$$

(ii) The lower Dini derivative at $x \in U$, in the direction $y$, is given by

$$
f^{-}(x ; y) \equiv \liminf _{\lambda \rightarrow 0^{+}} \frac{f(x+\lambda y)-f(x)}{\lambda} .
$$

(iii) The Clarke generalized directional derivative at $x \in U$, in the direction $y$, is given by

$$
f^{0}(x ; y) \equiv \limsup _{\substack{z \rightarrow x \\ \lambda \rightarrow 0^{+}}} \frac{f(z+\lambda y)-f(z)}{\lambda} .
$$

* Received by the editors January 26, 1996; accepted for publication (in revised form) February $21,1997$.

http://www.siam.org/journals/siopt/8-2/29783.html

${ }^{\dagger}$ CECM, Department of Mathematics and Statistics, Simon Fraser University, Burnaby, BC V5A 1S6, Canada (jborwein@cecm.sfu.ca). The research of this author was supported by NSERC and the Shrum Endowment at Simon Fraser University.

${ }^{\ddagger}$ Department of Mathematics, The University of Auckland, Private Bag 92019, Auckland, New Zealand (moors@math.auckland.ac.nz). The research of this author was supported by a New Zealand Science and Technology Post-Doctoral Fellowship. 
It is immediate from these three definitions that for each $x \in U$ and each $y \in X$,

$$
f^{-}(x ; y) \leq f^{+}(x ; y) \leq f^{0}(x ; y) .
$$

Let us now examine some notions of differentiability that are associated with locally Lipschitz functions. We say that a real-valued locally Lipschitz function $f$ is differentiable at $x$, in the direction $y$, if

$$
f^{\prime}(x ; y) \equiv \lim _{\lambda \rightarrow 0} \frac{f(x+\lambda y)-f(x)}{\lambda} \text { exists. }
$$

We say that $f$ is Gâteaux differentiable at $x$ if

$$
\nabla f(x)(y) \equiv \lim _{\lambda \rightarrow 0} \frac{f(x+\lambda y)-f(x)}{\lambda} \quad \text { exists }
$$

for each $y \in X$ and $\nabla f(x)$ is a continuous linear functional on $X$. In this paper we will also be interested in two slightly stronger notions of differentiability. A locally Lipschitz function $f$ is said to be strictly differentiable at $x$, in the direction $y$, if

$$
\lim _{\substack{z \rightarrow x \\ \lambda \rightarrow 0^{+}}} \frac{f(z+\lambda y)-f(z)}{\lambda} \text { exists, }
$$

and we say that $f$ is strictly differentiable at $x$, if $f$ is strictly differentiable at $x$, in every direction $y \in X$. Let us recall that a function $f$ is strictly differentiable at $x$, in the direction $y$, if and only if

$$
f^{0}(x ; y)=f^{\prime}(x ; y)=-f^{0}(x ;-y) .
$$

In addition to the various notions of differentiability we will also need the notion of a "null" set. Let $(X,\|\cdot\|)$ be a Banach space. We will call a Borel subset $N \subseteq X$ a Haar-null set if there exists a (not necessarily unique) Radon probability measure $p$ on $X$ such that $p(x+N)=0$ for each $x \in X$. (In this case, we call $p$ a test measure for $N$.)

More generally, we say that a subset $N \subseteq X$ is a Haar-null set if it is contained in a Borel Haar-null set (see [4] for a slightly more general definition of Haar-null). Further, we will say that a property $P$ holds almost everywhere if $\{x \in X: P(x)$ is not true $\}$ is a Haar-null set. The Haar-null sets are closed under translation and countable unions. Moreover, no nonempty open subset is Haar-null [4]. In finite dimensions, the Haar-null sets coincide with the Lebesgue-null sets.

A further important class of sets that we will need to consider is the following. Let $U$ be a nonempty open subset of a Banach space $X$. Then a Borel subset $S$ of $U$ is $1-D$ almost everywhere in $U$, in the direction $y$, if for each $x \in U$

$$
\mu(\{t \in R: x+t y \in U \quad \text { and } \quad x+t y \notin S\})=0 .
$$

(Here, and later, $\mu$ will denote the Lebesgue measure on $R$.)

For us, the most important example of a $1-D$ almost everywhere set is the following.

Proposition 1.1 (see [8, Remark 2.4]). Let $f$ be a real-valued locally Lipschitz function defined on a nonempty open subset $U$ of a Banach space $X$. Then for each $y \in X, D_{y} \equiv\left\{x \in U: f^{\prime}(x ; y)\right.$ exists $\}$ is $1-D$ almost everywhere in $U$, in the direction $y$. 
Clearly, if $S$ is $1-D$ almost everywhere in $U$, for some direction $y$, then $U \backslash S$ is a Haar-null set (see Proposition 2.2 in [4] if details are desired).

In [3] the present authors said that a real-valued locally Lipschitz function $f$ defined on a nonempty open subset $U$ of a Banach space $X$ is essentially smooth on $U$ if for each $y \in X$

$$
\left\{x \in U: f^{0}(x ; y) \neq-f^{0}(x ;-y)\right\} \text { is a Haar-null subset of } X
$$

and they denoted by $\mathcal{S}_{e}(U)$ the family of all real-valued essentially smooth locally Lipschitz functions defined on $U$.

On a few occasions we will need to consider vector-valued functions. Let $U$ be a nonempty open subset of a Banach space $X$ and let $V$ be a nonempty open subset of $R^{m}$. If $f: U \rightarrow V$ is defined by

$$
f(x) \equiv\left(f_{1}(x), f_{2}(x), \ldots, f_{m}(x)\right) \quad \text { with } f_{j}: U \rightarrow R,
$$

then we say that the vector-valued function $f$ is essentially smooth on $U$ if $f_{j} \in \mathcal{S}_{e}(U)$ for each $1 \leq j \leq m$, and in this case we write $f \in \mathcal{S}_{e}(U ; V)$. In addition, we will say that $f$ is strictly differentiable at $x \in U$, in the direction $y$, if

$$
f_{j}^{0}(x ; y)=-f_{j}^{0}(x ;-y) \quad \text { for each } 1 \leq j \leq m .
$$

Let us now return to the purpose of this paper which is, as mentioned earlier, to show that the family of functions $\mathcal{S}_{e}(U)$ possesses very strong closure properties. A first but naive guess might be that if $f_{1}, f_{2}, \ldots, f_{m} \in \mathcal{S}_{e}(U)$ and $g \in \mathcal{S}_{e}\left(R^{m}\right)$, then $g \circ f \in \mathcal{S}_{e}(U)$, where $f \equiv\left(f_{1}, f_{2}, \ldots, f_{m}\right)$. However, the following example shows that in general this is not true (when $m \geq 2$ ).

Example 1.1. Let $X$ be a Banach space, and let $C$ be a Cantor subset of $R$ with positive Lebesgue measure. Define the functions $f_{1}, \ldots, f_{m}$ on $X$ by $f_{m} \in X^{*} \backslash\{0\}$ and $f_{j} \equiv 0$ for each $1 \leq j<m$. Further, we define $d_{C}: R \rightarrow R$ by $d_{C}(t) \equiv \operatorname{dist}(t, C)$ and $g: R^{m} \rightarrow R$ by $g\left(x_{1}, x_{2}, \ldots, x_{m}\right) \equiv \operatorname{dist}\left(\left(x_{1}, x_{2}, \ldots, x_{m}\right),\{0\} \times\{0\} \times \cdots \times C\right)$ (here the distance is with respect to the Euclidean norm on $R^{m}$ ). Clearly, each $f_{j}$ is strictly differentiable on $X$. Moreover, by Theorem 6.2 in [3] we have that $g \in \mathcal{S}_{e}\left(R^{m}\right)$. We claim that $g \circ f \notin \mathcal{S}_{e}(X)$, where $f \equiv\left(f_{1}, f_{2}, \ldots, f_{m}\right)$. To see this, observe that $g \circ f(x)=d_{C}\left(f_{m}(x)\right)$. Now, it is standard that $d_{C}$ is not strictly differentiable at any point of $C$. Hence, it follows that $g \circ f$ is not strictly differentiable at any point of $f_{m}^{-1}(C)$, which is not a Haar-null set (see Theorem 2.3 in [4]). It now follows that $g \circ f \notin \mathcal{S}_{e}(X)$.

Despite this example, $\mathcal{S}_{e}(U)$ does possess very strong closure properties.

2. A chain rule. In this section of the paper, we show that there is a large subclass of the essentially smooth functions, which is closed under composition. This class of functions is closely related to Valadier's saine functions [9]. Let $U$ be a nonempty open subset of $R^{m}$. We say that a real-valued locally Lipschitz function $f$ defined on $U$ is arcwise essentially smooth on $U$ if for each locally Lipschitz function $x \in \mathcal{S}_{e}((0,1) ; U)$,

$$
\begin{gathered}
\mu\left(\left\{t \in(0,1): f^{0}\left(x(t) ; x^{\prime}(t)\right) \neq-f^{0}\left(x(t) ;-x^{\prime}(t)\right)\right\}\right)=0 \\
\text { here, } x^{\prime}(t) \equiv\left(x_{1}^{\prime}(t), x_{2}^{\prime}(t), \ldots, x_{m}^{\prime}(t)\right) .
\end{gathered}
$$

(Valadier requires this to hold for all absolutely continuous arcs.) We shall denote by $\mathcal{A}_{e}(U)$ the family of all arcwise essentially smooth functions defined on $U$. 
Remark 2.1. It is easily seen that the definition of arcwise essential smoothness is unaffected by replacing the open set $(0,1)$ (given in the definition) by any other nonempty open subset of $R$.

PROPOSITION 2.1. For each nonempty open subset $U$ of $R^{m} \mathcal{A}_{e}(U) \subseteq \mathcal{S}_{e}(U)$.

Proof. Consider $f \in \mathcal{A}_{e}(U)$. For each fixed $y \in S\left(R^{m}\right)$ (the unit sphere in $\left.R^{m}\right)$ we will show that the $G_{\delta}$ set, $S_{y} \equiv\left\{x \in U: f^{0}(x ; y)=-f^{0}(x ;-y)\right\}$ is $1-D$ almost everywhere in $U$, in the direction $y$. To this end, consider $x_{0} \in U$. Let $U_{0} \equiv\left\{t \in R: x_{0}+t y \in U\right\}$. Clearly $U_{0}$ is nonempty and open. Now, consider the mapping $x: U_{0} \rightarrow U$ defined by $x(t) \equiv x_{0}+t y$. It is obvious that $x \in S_{e}\left(U_{0} ; U\right)$. We may now perform a little set arithmetic.

$$
\begin{aligned}
& \left\{t \in R: x_{0}+t y \in U \text { and } x_{0}+t y \notin S_{y}\right\} \\
= & \left\{t \in U_{0}: f^{0}\left(x(t) ; x^{\prime}(t)\right) \neq-f^{0}\left(x(t) ;-x^{\prime}(t)\right)\right\}
\end{aligned}
$$

and so, $\mu\left(\left\{t \in R: x_{0}+t y \in U\right.\right.$ and $\left.\left.x_{0}+t y \notin S_{y}\right\}\right)=0$, which shows that $S_{y}$ is $1-D$ almost everywhere in $U$, in the direction $y$. Hence, $U \backslash S_{y}$ is a Haar-null (Lebesgue-null) set.

Proposition 2.2 ([3, Theorem 4.4]). If $U$ is a nonempty open subset of $R$, then $\mathcal{A}_{e}(U)=\mathcal{S}_{e}(U)$.

Our next task is to show that the class of functions $\mathcal{A}_{e}(U)$ is reasonably large, above and beyond the obvious observation that $\mathcal{A}_{e}(U)$ contains all the $C^{1}$ functions defined on $U$.

LEMMA 2.1. Let $U$ be a nonempty open subset of $R^{m}$, and let $f$ be a real-valued locally Lipschitz function defined on $U$. Then $f \in \mathcal{A}_{e}(U)$ if and only if for each $x \in \mathcal{S}_{e}((0,1) ; U)$

$$
\mu\left(\left\{t \in(0,1): f^{0}\left(x(t) ; x^{\prime}(t)\right)=f^{\prime}\left(x(t) ; x^{\prime}(t)\right)\right\}\right)=1 .
$$

Proof. Let $x \in S_{e}((0,1) ; U)$. We need to show that

$$
\mu\left(\left\{t \in(0,1): f^{0}\left(x(t) ; x^{\prime}(t)\right)=-f^{0}\left(x(t) ;-x^{\prime}(t)\right)\right\}\right)=1 .
$$

Consider the mapping $y:(0,1) \rightarrow U$, defined by $y(t) \equiv x(1-t)$. Note that almost everywhere $y^{\prime}(t)=-x^{\prime}(1-t)$.

Let $E_{1} \equiv\left\{t \in(0,1): f^{0}\left(x(t) ; x^{\prime}(t)\right)=f^{\prime}\left(x(t) ; x^{\prime}(t)\right)\right\}$ and $E_{2} \equiv\{t \in(0,1)$ : $\left.-f^{0}\left(x(t) ;-x^{\prime}(t)\right)=f^{\prime}\left(x(t) ; x^{\prime}(t)\right)\right\}$. Clearly, $f^{0}\left(x(t) ; x^{\prime}(t)\right)=-f^{0}\left(x(t) ;-x^{\prime}(t)\right)$ on $E_{1} \cap E_{2}$, so it remains to show that $\mu\left(E_{2}\right)=1$.

Now, by the hypothesis, $E_{3} \equiv\left\{t \in(0,1): f^{\prime}\left(y(t) ; y^{\prime}(t)\right)=f^{0}\left(y(t) ; y^{\prime}(t)\right)\right\}$ has measure one. On the other hand, $E_{2}=h\left(E_{3}\right)$, where $h(t) \equiv 1-t$. Therefore, $\mu\left(E_{2}\right)=1$. The converse is obvious.

The next few lemmas are quite standard, but we include them for the sake of completeness.

LEMMA 2.2. Let $U$ be a nonempty open subset of a Banach space $X$ and let $f \equiv\left(f_{1}, f_{2}, \ldots, f_{m}\right)$ be a locally Lipschitz mapping from $U$ into $R^{m}$. Furthermore, let $g$ be a real-valued locally Lipschitz function defined on a nonempty open subset $V$ of $R^{m}$ which contains $f(U)$.

If $f$ is differentiable at some point $x_{0}$, in the direction $y$, and either

(i) $(g \circ f)^{\prime}\left(x_{0} ; y\right)$ exists or (ii) $g^{\prime}\left(f\left(x_{0}\right) ; f^{\prime}\left(x_{0} ; y\right)\right)$ exists, then

$$
(g \circ f)^{\prime}\left(x_{0} ; y\right)=g^{\prime}\left(f\left(x_{0}\right) ; f^{\prime}\left(x_{0} ; y\right)\right) .
$$


Remark 2.2. From Lemma 2.2 and Proposition 1.1 we see that for each fixed $y \in X,\left\{x \in U:(g \circ f)^{\prime}(x ; y)=g^{\prime}\left(f(x) ; f^{\prime}(x ; y)\right)\right\}$ is $1-D$ almost everywhere in $U$, in the direction $y$.

LEMMA 2.3 ([4, Theorem 3.1]). Let $f$ be a real-valued locally Lipschitz function defined on a nonempty open subset $U$ of a Banach space $X$. If $U \backslash S$ is a Haar-null set, then for each $x \in U$

$$
f^{0}(x ; y)=\limsup _{\substack{z \rightarrow x \\ z \in S}} f^{+}(z ; y) \text { and }-f^{0}(x ;-y)=\liminf _{\substack{z \rightarrow x \\ z \in S}} f^{+}(z ; y) .
$$

Remark 2.3. From Lemma 2.3 we see that $f^{0}(x ; y)=-f^{0}(x ;-y)$ if and only if

$$
\lim _{\substack{z \rightarrow x \\ z \in S}} f^{+}(z ; y) \text { exists and equals } f^{\prime}(x ; y) \text {. }
$$

The following lemma encapsulates the heart of our chain rule.

LEMMA 2.4. Let $U$ be a nonempty open subset of a Banach space $X$, and let $f \equiv\left(f_{1}, f_{2}, \ldots, f_{m}\right)$ be a locally Lipschitz mapping from $U$ into $R^{m}$. Furthermore, let $g$ be a real-valued locally Lipschitz function defined on a nonempty open subset $V$ of $R^{m}$ which contains $f(U)$.

If $f$ is strictly differentiable at some point $x_{0} \in U$, in the direction $y$, and $g^{0}\left(f\left(x_{0}\right)\right.$; $\left.f^{\prime}\left(x_{0} ; y\right)\right)=-g^{0}\left(f\left(x_{0}\right) ;-f^{\prime}\left(x_{0} ; y\right)\right)$, then $g \circ f$ is strictly differentiable at $x_{0}$, in the direction $y$.

Proof. Let $D \equiv\left\{x \in U:(g \circ f)^{\prime}(x ; y)=g^{\prime}\left(f(x) ; f^{\prime}(x ; y)\right)\right\}$. It follows from Remark 2.2 that $D$ is $1-D$ almost everywhere in $U$, in the direction $y$.

(i) Let us first observe that $x_{0} \in D$. From Remark 2.3 we see that to show $(g \circ f)^{0}\left(x_{0} ; y\right)=-(g \circ f)^{0}\left(x_{0} ;-y\right)$ we need only show that

$$
\lim _{\substack{z \rightarrow x_{0} \\ z \in D}}(g \circ f)^{\prime}(z ; y) \text { exists and equals }(g \circ f)^{\prime}\left(x_{0} ; y\right) .
$$

So suppose $\epsilon>0$. By the continuity of the mapping, $x \rightarrow g^{+}\left(x ; f^{\prime}\left(x_{0} ; y\right)\right)$, at $f\left(x_{0}\right)$ there exists an open neighborhood $W_{1}$ of $x_{0}$ such that

$$
\left|g^{+}\left(f(z) ; f^{\prime}\left(x_{0} ; y\right)\right)-g^{\prime}\left(f\left(x_{0}\right) ; f^{\prime}\left(x_{0} ; y\right)\right)\right|<\epsilon / 2
$$

for each $z \in W_{1}$. Now, as $g$ is locally Lipschitz there exists an open neighborhood $W_{2}$ of $x_{0}$ and a constant $M>0$ such that

$$
\left|g^{\prime}\left(f(z) ; f^{\prime}(z ; y)\right)-g^{+}\left(f(z) ; f^{\prime}\left(x_{0} ; y\right)\right)\right| \leq M\left\|f^{\prime}(z ; y)-f^{\prime}\left(x_{0} ; y\right)\right\|
$$

for each $z \in D \cap W_{2}$. On the other hand, $x \rightarrow f^{+}(x ; y)$ is continuous at $x_{0}$; therefore, there exists an open neighborhood $W_{3}$ of $x_{0}$ such that

$$
\left\|f^{\prime}\left(x_{0} ; y\right)-f^{+}(x ; y)\right\|<\epsilon / 2 M
$$

for each $x \in W_{3}$. Hence we have that for each $x \in D \cap W_{1} \cap W_{2} \cap W_{3}$,

$$
\begin{aligned}
\left|(g \circ f)^{\prime}\left(x_{0} ; y\right)-(g \circ f)^{\prime}(x ; y)\right|= & \left|g^{\prime}\left(f\left(x_{0}\right) ; f^{\prime}\left(x_{0} ; y\right)\right)-g^{\prime}\left(f(x) ; f^{\prime}(x ; y)\right)\right| \\
\leq & \left|g^{\prime}\left(f\left(x_{0}\right) ; f^{\prime}\left(x_{0} ; y\right)\right)-g^{+}\left(f(x) ; f^{\prime}\left(x_{0} ; y\right)\right)\right| \\
& +\left|g^{+}\left(f(x) ; f^{\prime}\left(x_{0} ; y\right)\right)-g^{\prime}\left(f(x) ; f^{\prime}(x ; y)\right)\right| \\
\leq & \epsilon / 2+\epsilon / 2=\epsilon .
\end{aligned}
$$


Therefore,

$$
\lim _{\substack{z \rightarrow x_{0} \\ z \in D}}(g \circ f)^{\prime}(z ; y)=(g \circ f)^{\prime}\left(x_{0} ; y\right) .
$$

We need a few more definitions before we can show that $\mathcal{A}_{e}(U)$ contains a significant class of functions (above and beyond the $C^{1}$ functions). Let $f$ be a real-valued locally Lipschitz function defined on a nonempty open subset $U$ of a Banach space $X$. Then $f$ is upper semismooth (lower semismooth) at a point $x \in U$, in the direction $y$, if

$$
f^{+}(x ; y) \geq \limsup _{\substack{t \rightarrow 0^{+} \\ y^{\prime} \rightarrow y}} f^{+}\left(x+t y^{\prime} ; y\right) \quad\left(f^{-}(x ; y) \leq \liminf _{\substack{t \rightarrow 0^{+} \\ y^{\prime} \rightarrow y}} f^{-}\left(x+t y^{\prime} ; y\right)\right)
$$

Moveover, we say that $f$ is semismooth at a point $x \in U$, in the direction $y$, if

$$
\limsup _{\substack{t \rightarrow 0^{+} \\ y^{\prime} \rightarrow y}} f^{+}\left(x+t y^{\prime} ; y\right)=f^{+}(x ; y)=f^{-}(x ; y)=\liminf _{\substack{t \rightarrow 0^{+} \\ y^{\prime} \rightarrow y}} f^{-}\left(x+t y^{\prime} ; y\right) .
$$

To see that this terminology is justified we direct the reader's attention to Corollary 6.3 in [7]. If $X$ is finite dimensional then we say that $f$ is arcwise essentially upper semismooth (arcwise essentially lower semismooth) on $U$ if for each $x \in \mathcal{S}_{e}((0,1) ; U)$ we have that $f$ is upper (lower) semismooth at $x(t)$ in the direction $x^{\prime}(t)$ for almost all $t \in(0,1)$.

The following technical result allows us to perform only "unilateral" analysis in our main theorem.

THEOREM 2.1. Let $f$ be a real-valued locally Lipschitz function defined on a nonempty open subset $U$ of a finite dimensional Banach space $X$. Then $f$ is arcwise essentially upper semismooth (arcwise essentially lower semismooth) on $U$ if and only if $f \in \mathcal{A}_{e}(U)$.

Proof. Suppose that $f$ is arcwise essentially upper semismooth on $U$ (the proof for the case when $f$ is arcwise essentially lower semismooth on $U$ is similar). Let $x \in \mathcal{S}_{e}((0,1) ; U)$. Consider the mappings $P:(0,1) \rightarrow R$ and $T:(0,1) \rightarrow R$ defined by

$$
P(t) \equiv f^{0}\left(x(t) ; x^{+}(t)\right) \quad \text { and } \quad T(t) \equiv \limsup _{t^{\prime} \rightarrow t^{+}} P\left(t^{\prime}\right) .
$$

Clearly, $T(t) \leq P(t)$ almost everywhere in $(0,1)$. Indeed, $T(t) \leq P(t)$ at each point of $S \equiv\{t \in(0,1): x$ is strictly differentiable at $t\}$. We claim that $E \equiv\{t \in S: T(t)<$ $P(t)\}$ is countable (and hence $T(t)=P(t)$ almost everywhere in $(0,1)$ ). Without loss of generality let us assume that $E \neq \emptyset$. In this case, we define $s: E \rightarrow Q^{2}$ by $s(t) \equiv\left(r_{1}, r_{2}\right)$, where $r_{1} \in(T(t), P(t)) \cap Q$ and $r_{2} \in(t, 1) \cap Q$ is chosen so that $\sup \left\{P\left(t^{\prime}\right): t<t^{\prime}<r_{2}\right\}<r_{1}$. It is easy to see that $s$ is 1 -to-1 and so $E$ must be at most countable (here $Q$ denotes the rational numbers). We now show that $P(t)=f^{+}\left(x(t) ; x^{\prime}(t)\right)$ at almost every point of $S \backslash E$. To prove this, consider an arbitrary point $t_{0} \in S \backslash E$ where $f$ is upper semismooth at $x(t)$ in the direction $x^{\prime}(t)$. Let $\epsilon>0$ be fixed but arbitrary. We will show that $T\left(t_{0}\right) \leq f^{+}\left(x\left(t_{0}\right) ; x^{\prime}\left(t_{0}\right)\right)+\epsilon$. Note that without loss of generality we may assume that $x^{\prime}\left(t_{0}\right) \neq 0$. Now, since $f$ is upper semismooth at $x\left(t_{0}\right)$ in the direction $x^{\prime}\left(t_{0}\right)$, there exists a $0<\delta_{1}$ such that

$$
f^{+}\left(z ; x^{\prime}\left(t_{0}\right)\right) \leq f^{+}\left(x\left(t_{0}\right) ; x^{\prime}\left(t_{0}\right)\right)+\epsilon / 2
$$


for each $z$ in the nonempty open subset $V$, where $V$ equals

$$
V \equiv\left\{z \in U: z=x\left(t_{0}\right)+t y, 0<t<\delta_{1} \text { and }\left\|y-x^{\prime}\left(t_{0}\right)\right\|<\delta_{1}\right\} .
$$

Let

$$
E(\lambda) \equiv \frac{x\left(t_{0}+\lambda\right)-x\left(t_{0}\right)}{\lambda}-x^{\prime}\left(t_{0}\right) \quad \text { for } 0<\lambda<\left(1-t_{0}\right) .
$$

Since $x$ is Gâteaux differentiable at $t_{0}$, there exists a $\delta_{2} \in\left(0, \delta_{1}\right)$ such that $\|E(\lambda)\|_{\infty}<$ $\delta_{1}$ for each $\lambda \in\left(0, \delta_{2}\right)$. Therefore, for each $\lambda \in\left(0, \delta_{2}\right)$

$$
x\left(t_{0}+\lambda\right)=x\left(t_{0}\right)+\lambda\left(x^{\prime}\left(t_{0}\right)+E(\lambda)\right) \in V,
$$

and so, $f^{0}\left(x\left(t_{0}+\lambda\right) ; x^{\prime}\left(t_{0}\right)\right) \leq f^{+}\left(x\left(t_{0}\right) ; x^{\prime}\left(t_{0}\right)\right)+\epsilon / 2$. Now, as $f$ is locally Lipschitz there exists a constant $M>0$ and a $\delta_{3} \in\left(0, \delta_{2}\right)$ such that

$$
\left|P\left(t_{0}+\lambda\right)-f^{0}\left(x\left(t_{0}+\lambda\right) ; x^{\prime}\left(t_{0}\right)\right)\right| \leq M\left\|x^{+}\left(t_{0}+\lambda\right)-x^{\prime}\left(t_{0}\right)\right\|<\epsilon / 2
$$

for each $\lambda \in\left(0, \delta_{3}\right)$. From this we get that $P\left(t_{0}+\lambda\right) \leq f^{+}\left(x\left(t_{0}\right) ; x^{\prime}\left(t_{0}\right)\right)+\epsilon$ for each $\lambda \in\left(0, \delta_{3}\right)$, and so, $T\left(t_{0}\right) \leq f^{+}\left(x\left(t_{0}\right) ; x^{\prime}\left(t_{0}\right)\right)+\epsilon$. However, since $\epsilon$ was arbitrary, $P\left(t_{0}\right)=T\left(t_{0}\right) \leq f^{+}\left(x\left(t_{0}\right), x^{\prime}\left(t_{0}\right)\right)$. The result now follows from Lemma 2.1. The converse is obvious.

Next we show that the family of functions $\mathcal{A}_{e}(U)$ enjoys quite remarkable closure properties.

THEOREM 2.2. Let $U$ be a nonempty open subset of $R^{n}$, and suppose that $f_{1}, f_{2}, \ldots, f_{m} \in \mathcal{A}_{e}(U)$. Let $g$ be a real-valued locally Lipschitz function defined on a nonempty open subset $V$ of $R^{m}$, which contains $f(U)$, where $f \equiv\left(f_{1}, f_{2}, \ldots, f_{m}\right)$. Then $g \circ f \in \mathcal{A}_{e}(U)$ whenever $g \in \mathcal{A}_{e}(V)$.

Proof. Let $x \in \mathcal{S}_{e}((0,1) ; U)$, and define $z:(0,1) \rightarrow V$ by $z(t) \equiv f(x(t))$. Our first task is to show that $z \in \mathcal{S}_{e}((0,1) ; V)$. However, this follows immediately from Lemma 2.4 and the fact that $f \in \mathcal{A}_{e}(U)$.

Let $S_{f} \equiv\left\{t \in(0,1): f_{j}^{0}\left(x(t) ; x^{\prime}(t)\right)=-f_{j}^{0}\left(x(t) ;-x^{\prime}(t)\right)\right.$ for $\left.1 \leq j \leq m\right\}$ and $S_{g} \equiv\left\{t \in(0,1): g^{0}\left(z(t) ; z^{\prime}(t)\right)=-g^{0}\left(z(t) ;-z^{\prime}(t)\right)\right\}$. Now, define $S \equiv S_{f} \cap S_{g}$. Clearly, $\mu(S)=1$.

We claim that $(g \circ f)^{0}\left(x(t) ; x^{\prime}(t)\right)=-(g \circ f)^{0}\left(x(t) ;-x^{\prime}(t)\right)$ at each point of $S$. To this end, consider any point $t_{0} \in S$. Let us first observe that if $x^{\prime}\left(t_{0}\right)=0$, then we are done. So suppose that $x^{\prime}\left(t_{0}\right) \neq 0$. Set $x_{0} \equiv x\left(t_{0}\right)$ and $y \equiv x^{\prime}\left(t_{0}\right)$. Then we have that $f$ is strictly differentiable at $x_{0}$, in the direction $y$, and $g^{0}\left(f\left(x_{0}\right) ; f^{\prime}\left(x_{0} ; y\right)\right)=$ $-g^{0}\left(f\left(x_{0}\right) ;-f^{\prime}\left(x_{0} ; y\right)\right)$ since $t_{0} \in S_{g}$.

Therefore from Lemma 2.4, $g \circ f$ is strictly differentiable at $x_{0}$, in the direction $y$; that is, $(g \circ f)^{0}\left(x\left(t_{0}\right) ; x^{\prime}\left(t_{0}\right)\right)=-(g \circ f)^{0}\left(x\left(t_{0}\right) ;-x^{\prime}\left(t_{0}\right)\right)$.

On relaxing the hypotheses on $f_{1}, f_{2}, \ldots, f_{m}$ we may still recover a satisfactory and important theorem.

THEOREM 2.3. Let $U$ be a nonempty open subset of a Banach space $X$, and let $f \equiv\left(f_{1}, f_{2}, \ldots, f_{m}\right) \in \mathcal{S}_{e}(U ; V)$, where $V$ is any nonempty open subset of $R^{m}$ that contains $f(U)$. Then $g \circ f \in \mathcal{S}_{e}(U)$ whenever $g \in \mathcal{A}_{e}(V)$.

Proof. It suffices to show that for each $y \in S(X),(g \circ f)^{0}(x ; y)=-(g \circ f)^{0}(x ;-y)$ almost everywhere in $U$. So fix $y \in S(X)$. Let $D$ be the $\left(G_{\delta}\right)$ set of all points in $U$ where $f_{j}^{0}(x ; y)=-f_{j}^{0}(x ;-y)$ for each $1 \leq j \leq m$, and let $P_{y} \equiv\{x \in U$ : $\left.(g \circ f)^{0}(x ; y)=-(g \circ f)^{0}(x ;-y)\right\}$. Clearly $P_{y}$ is a Borel set; in fact, $P_{y}$ is a $G_{\delta}$ set. 
Let $H$ be any closed hyperplane in $X$ such that $y \notin H$. Now consider the isomorphism $T: H \times R \rightarrow X$ defined by $T(h, t) \equiv h+t y$. Let

$$
H_{D} \equiv\{h \in H: \mu(\{t \in R: T(h, t) \in U \backslash D\})=0\} .
$$

By Theorem 2.3 in [4] we see that $H \backslash H_{D}$ is a Haar-null set in $H$, since $U \backslash D$ is a Haarnull set in $X$. To show that $U \backslash P_{y}$ is a Haar-null set in $X$ it suffices (also because of Theorem 2.3 in [4]) to show that for each $h \in H_{D}, \mu\left(\left\{t \in R: T(h, t) \in U \backslash P_{y}\right\}\right)=0$.

To this end, consider $h_{0} \in H_{D}$. Let $U_{h_{0}} \equiv\left\{t \in R: T\left(h_{0}, t\right) \in U\right\}$. If $U_{h_{0}}=\emptyset$ then we are done, so let us suppose that $U_{h_{0}} \neq \emptyset$. Define $z: U_{h_{0}} \rightarrow V$ by $z(t) \equiv f\left(h_{0}+t y\right)$. Since $h_{0} \in H_{D}, z \in \mathcal{S}_{e}\left(U_{h_{0}} ; V\right)$. Let

$$
D_{f} \equiv\left\{t \in U_{h_{0}}: f_{j}^{0}\left(h_{0}+t y ; y\right)=-f_{j}^{0}\left(h_{0}+t y ;-y\right) \text { for all } 1 \leq j \leq m\right\}
$$

and

$$
D_{g} \equiv\left\{t \in U_{h_{0}}: g^{0}\left(z(t) ; z^{\prime}(t)\right)=-g^{0}\left(z(t) ;-z^{\prime}(t)\right)\right\} .
$$

Now define $D_{0} \equiv D_{f} \cap D_{g}$. Clearly, $\lambda\left(U_{h_{0}} \backslash D_{0}\right)=0$. We claim that

$$
(g \circ f)^{0}\left(h_{0}+t y ; y\right)=-(g \circ f)^{0}\left(h_{0}+t y:-y\right)
$$

at each point $t \in D_{0}$. To see this, consider an arbitrary point $t_{0} \in D_{0}$. Set $x_{0} \equiv$ $h_{0}+t_{0} y$, then $g^{0}\left(f\left(x_{0}\right) ; f^{\prime}\left(x_{0} ; y\right)\right)=-g^{0}\left(f\left(x_{0}\right) ;-f^{\prime}\left(x_{0} ; y\right)\right)$ as $t_{0} \in D_{g}$ and $f_{j}^{0}\left(x_{0} ; y\right)=$ $-f_{j}^{0}\left(x_{0} ;-y\right)$ for each $1 \leq j \leq m$ as $t_{0} \in D_{f}$. It now follows from Lemma 2.4 that $(g \circ f)^{0}\left(x_{0} ; y\right)=-(g \circ f)^{0}\left(x_{0} ;-y\right)$. This completes the proof.

Remark 2.4. It follows from Theorem 2.3 that the distance function $g$ defined in Example 1.1 lies in $\mathcal{S}_{e}\left(R^{m}\right) \backslash \mathcal{A}_{e}\left(R^{m}\right),(m \geq 2)$. However, by translation and dilation (of this example) one can show that for every nonempty open subset $U$ of $R^{m}(m \geq 2)$, $\mathcal{A}_{e}(U)$ is a proper subset of $\mathcal{S}_{e}(U)$.

COROLlaRY 2.1. Let $U$ be a nonempty open subset of $R^{m}$. Then $\mathcal{A}_{e}(U)$ and $\mathcal{S}_{e}(U)$ are function algebras and vector lattices containing all the convex and $C^{1}$ functions.

Let us also observe that the class of functions $\mathcal{A}_{e}\left(R^{m}\right)$ contains many distance functions. Indeed, one can show that if $C$ is a closed regular subset of $R^{m}$ [6], then the distance function generated by this set and any smooth norm on $R^{m}$ is in this class. To see this, we need to make the following observations:

(i) For any norm on $R^{m}$, the distance function $d_{C}$ is regular at each point of $C$, see [1] or [6].

(ii) If the norm is smooth on $R^{m}$, then $-d_{C}$ is regular on $R^{n} \backslash C$, see [2].

We can now define a class of closed subsets of $R^{m}$ as follows. A closed subset $C$ of $R^{m}$ is arcwise essentially smooth if for each $x \in \mathcal{S}_{e}\left((0,1) ; R^{m}\right)$ the set

$$
\left\{t \in(0,1): x(t) \in C \text { and } x^{\prime}(t) \in K_{C}(x(t)) \backslash T_{C}(x(t))\right\}
$$

has measure zero. Here $K_{C}(x)$ denotes the contingent cone of $C$ at $x$ and $T_{C}(x)$ denotes the Clarke tangent cone of $C$ at $x$. Recall that $C$ is regular at $x$ if $K_{C}(x)=$ $T_{C}(x)[6]$. Note that all sets that are regular except on a countable set are arcwise essentially smooth. Thus, all closed convex sets and all smooth manifolds are arcwise essentially smooth.

Proposition 2.3. Let $\|\cdot\|$ be a smooth norm on $R^{m}$, and let $C$ be a nonempty closed subset of $R^{m}$. Then the distance function generated by the norm $\|\cdot\|$ and the set $C$ is a member of $\mathcal{A}_{e}\left(R^{m}\right)$ if and only if $C$ is arcwise essentially smooth. 
It is immediate from this proposition and Theorem 2.2 that, unlike the family of regular sets, the family of arcwise essentially smooth sets is closed under finite unions. We should also observe that from Proposition 2.1 it follows that the boundary of every arcwise essentially smooth set is a Lebesgue-null set. We also note that the set $\{0\} \times\{0\} \times \cdots \times C$ in Example 1.1 is an example of a null set that is not arcwise essentially smooth. Finally, observe that we may now provide general conditions to ensure that exact penalty functions of the form

$$
f(x)+K d_{C}(x)
$$

will be in $\mathcal{A}_{e}(U)$ or in $\mathcal{S}_{e}(U)$.

\section{REFERENCES}

[1] J. M. Borwein And M. Fabian, A note on regularity of sets and of distance functions in Banach space, J. Math. Anal. Appl., 182 (1994), pp. 566-560.

[2] J. M. Borwein, S. P. Fitzpatrick, and J. R. Giles, The differentiability of real functions on normed linear spaces using generalized subgradients, J. Math. Anal. Appl., 128 (1987), pp. $512-534$.

[3] J. M. Borwein And W. B. Moors, Essentially smooth Lipschitz functions, J. Funct. Anal., 149 (1997), pp. 305-351.

[4] J. M. BorweIN AND W. B. Moors, Null sets and essentially smooth Lipschitz functions, SIAM J. Optim., 8 (1998), pp. 309-323.

[5] J. P. R. Christensen, On sets of Haar measure zero in Abelian groups, Israel J. Math., 13 (1972), pp. 255-260.

[6] F. H. Clarke, Optimization and Nonsmooth Analysis, John Wiley, New York, 1971.

[7] R. Correa And A. Jofre, Tangentially continuous directional derivatives in nonsmooth analysis, JOTA, 61 (1989), pp. 1-21.

[8] W. B. Moors, A characterisation of minimal subdifferential mappings of locally Lipschitz functions, Set-Valued Anal., 3 (1995), pp. 129-141.

[9] M. VAlADIER, Entrainement unilateral, lignes de descente, fouctions Lipshitziennes non pathologiques, C. R. Acad. Sci. Paris 308, Series I, 1989, pp. 241-244. 